\title{
Disponibilidade alimentar para famílias residentes na zona rural: situação de segurança ou insegurança alimentar e nutricional
}

\author{
Luiza Veloso Dutra ${ }^{1}$, Luiza Monteiro Souza ${ }^{2}$, Ricardo Henrique Silva Santos ${ }^{3}$ e Silvia Eloiza \\ Priore $^{4}$
}

O objetivo deste trabalho foi analisar os alimentos disponíveis para consumo, sua origem e a situação de (in)segurança alimentar e nutricional de famílias da Zona da Mata de Minas Gerais. O estudo foi realizado com 10 famílias, totalizando 40 pessoas, das quais foram avaliados o peso e a estatura. Para investigação da disponibilidade de alimentos foi utilizado questionário contendo lista de procedência e quantidade mensal destes nos últimos 30 dias. Os resultados indicaram $62,5 \%$ de indivíduos eutróficos, 30\% com excesso de peso e 7,5\% com baixo peso, sendo a maioria dos casos de inadequação em mulheres. A média da disponibilidade calórica supre 156,5\% da necessidade calórica das famílias, classificando todas em segurança. Das calorias disponíveis, $40,5 \%$ provinham da produção para o autoconsumo e a participação dos macronutrientes indicou carboidratos $50,6 \%$, proteínas $11,5 \%$ e lipídeos 37,8\%. Apesar da classificação de segurança, o excesso de calorias disponíveis é preocupante, pois a totalidade da sua ingestão pode agravar o quadro atual de $30 \%$ da população com excesso de peso e a qualidade dos alimentos disponíveis não apresentou a adequação esperada, tornando essas famílias mais propícias a apresentarem insegurança alimentar. É importante incentivar a produção para autoconsumo, pois esta pode contribuir para o alcance da segurança alimentar e nutricional das famílias.

Palavras-chave: agricultura familiar, estado nutricional, segurança alimentar e nutricional.

\section{Food available for families living in rural area: the state of food security or insecurity}

This study aimed to analyze the foods available for consumption, their origin and status Food Insecurity family farmers in the Zona da Mata of Minas Gerais. The study was conducted with 10 families totaling 40 people, which we assessed weight and height. To research the availability of food questionnaire was used containing list of source and monthly amount. The results indicated $62.5 \%$ of normal individuals, $30 \%$ overweight and $7.5 \%$ underweight, the majority cases of inadequacy in women. The average calorie availability supplies $156.5 \%$ of the caloric needs of families, all in Food Security ranking, according to the method of Smith. The calories available, $40.5 \%$ came from local production and participation of macronutrients indicated $50.6 \%$ carbohydrates, $11.52 \%$ protein and $37.88 \%$ lipid, indicating inadequate availability of lipids for the recommendation. Despite the classification of food security, the excess calories available is worrisome because their intake can aggravate the current situation in which $30 \%$ of the population was overweight and quality of food available did not show the

\footnotetext{
${ }_{1}$ Mestre em Agroecologia, Programa de Pós-Graduação em Agroecologia, Universidade Federal de Viçosa, MG. Correspondência: Rua Professor Padre Anchieta no 40, apto. 301 - Ramos - Viçosa - Minas Gerais - Brasil. CEP 36570-000. E-mail: luizavdutra@gmail.com

${ }_{2}^{2}$ Departamento de Fitotecnia, Universidade Federal de Viçosa, MG. E-mail: luiza.sz1@gmail.com

${ }^{3}$ Docente do Departamento de Fitotecnia, Universidade Federal de Viçosa, MG. E-mail: rsantos@ufv.br

${ }^{4}$ Docente do Departamento de Nutrição e Saúde, Universidade Federal de Viçosa, MG. E-mail: sepriore@ufv.br
} 
expected fitness, making these families more likely the present situation of food insecurity. It is important to encourage the production of food for own consumption since these can contribute substantially to the achievement of food and nutrition security of farmers and their families.

Key-words: family agriculture, nutritional status, food security.

\section{INTRODUÇÃO}

Considera-se no mundo todo, como um dos desafios do início do século XXI, a necessidade de superar a fome, que afeta grande contingente da população, num ambiente que apresenta capacidade de produção de alimentos muito desenvolvida [1]. A Organização das Nações Unidas para Alimentação e Agricultura [2] apontou, pela primeira vez em 15 anos, que o número de pessoas subnutridas no mundo diminuiu de 1,023 bilhão em 2009, para 925 milhões, em 2010.

O Conselho Nacional de Segurança Alimentar e Nutricional [3] define que a Segurança Alimentar e Nutricional "é o direito de todos ao acesso regular e permanente a alimentos de qualidade, em quantidade suficiente, sem comprometer o acesso a outras necessidades essenciais, tendo como base práticas alimentares promotoras de saúde, que respeitem a diversidade cultural e que sejam social, econômica e ambientalmente sustentáveis".

Contudo, a utilização do conceito de Segurança Alimentar e Nutricional (SAN) dá origem a diferentes interpretações. Países ricos, grandes produtores agrícolas, costumam alegar motivos de SAN para impor barreiras às importações e elevar artificialmente os preços dos alimentos. A maioria dos países pobres, governados por líderes populistas, utiliza-se desse conceito para tabelar preços e impor pesadas perdas aos produtores agrícolas com a finalidade de contentar os seus eleitores. Da mesma maneira, a SAN é invocada por interesses particulares para promover a destruição do meio ambiente ou mesmo a destruição dos hábitos culturais de um povo. Conforme registrado pela Organização para a Agricultura e a Alimentação (FAO) os alimentos podem estar disponíveis, mas não acessíveis à população, seja por problemas de renda ou devido a outros fatores como conflitos internos, ação de monopólios ou mesmo desvios, levando a Insegurança Alimentar e Nutricional [4,5].
Nesse contexto a agricultura familiar pode representar a ocupação socialmente mais equitativa do espaço agrário, bem como favorecer a valorização das dimensões social, ambiental e cultural da produção agroalimentar para autoconsumo, como é próprio da proposta da Segurança Alimentar e Nutricional [ø.

A produção para autoconsumo deveria gerar Segurança Alimentar e Nutricional aos agricultores e familiares, por meio da garantia de produção dos alimentos básicos, que integram a alimentação dos membros do grupo doméstico. Porém, o processo de mercantilização social e econômica da agricultura familiar leva alguns agricultores familiares a vulnerabilizarem a produção para autoconsumo, optando pela especialização produtiva, podendo chegar a situações de Insegurança Alimentar e Nutricional [7].

Além disso, na contemporaneidade, assiste-se à generalização de hábitos alimentares inadequados, inclusive em famílias da área rural, como consequência da atuação da indústria alimentícia, e devido ao comportamento dos consumidores, envoltos em rotinas profissionais ou por disporem de informações insuficientes e equivocadas sobre a alimentação. Proporciona-se, dessa forma, uma dieta desequilibrada, gerando situações de desnutrição ou de obesidade. Parcela da população mundial tem acesso diário e regular a gêneros alimentícios, entretanto fazem escolhas e combinações cujos efeitos, para o organismo, são o fornecimento insuficiente de determinados elementos essenciais para uma nutrição adequada, gerando a fome oculta, e a ingestão excessiva de outras substâncias, contribuindo para a incidência de sobrepeso, obesidade, hipertensão arterial, entre outras complicações associadas ao excesso de peso [1].

A agricultura familiar sustentável assume papel relevante no contexto do presente estudo, pois é responsável por grande parte da produção de alimentos no país, em especial para o consumo familiar [8]. O estudo da disponibilidade para o autoconsumo pode contribuir primeiro, para viabilizar a possibilidade de melhor interpretar as estatísticas referentes à renda agrícola do produtor familiar. Segundo, pode fornecer 
subsídios para criar situação de Segurança Alimentar e Nutricional, no meio rural, ao propiciar maior qualidade de alimentos in natura, frescos e habituais. A importância desse estudo está na contribuição ao tratar a produção para o autoconsumo como fonte geradora de Segurança Alimentar e Nutricional para os agricultores familiares. Neste sentido o objetivo deste trabalho foi analisar os alimentos disponíveis para consumo humano, sua origem e a situação de Segurança ou Insegurança Alimentar e Nutricional das famílias de agricultores familiares da Zona da Mata de Minas Gerais.

\section{METODOLOGIA}

O estudo foi realizado com quarenta pessoas pertencentes a dez famílias rurais, participantes do projeto (condição de inclusão) “Apoio à transição agroecológica de novas famílias no território da Serra do Brigadeiro, Minas Gerais" do Departamento de Fitotecnia da Universidade Federal de Viçosa que conta com a participação da Empresa de Assistência Técnica e Extensão Rural (EMATER - MG) no município de São Miguel do Anta, Minas Gerais. O projeto de apoio à transição agroecológica em sua fase inicial trabalhou com reduzido número de famílias, o que justifica o tamanho da amostra.

Segundo estimativa do Instituto Brasileiro de Geografia e Estatística [9] em 2009, São Miguel do Anta - MG, possuia 7.094 habitantes e de 1970 até 2000 o êxodo rural aumentou $21,56 \%$ equiparando as populações rural e urbana. O Produto Interno Bruto PIB per capita em 2007 foi de 5.014,00 reais e o agropecuário representava $36,53 \%$ do PIB bruto ficando atrás apenas de serviços, diferente de Minas Gerais e Brasil onde o agropecuário ocupa a terceira posição.

Os dados foram coletados entre os meses de março e junho de 2010.

\section{Avaliação da situação de Segurança Alimentar e Nutricional}

Utilizando-se inquérito domiciliar, aplicado em um dia por um único observador, os moradores informaram os alimentos disponíveis no domicilio referente aos últimos 30 dias. Foram relatadas as quantidades que cada família dispunha a partir da produção no domicílio para consumo, bem como alimentos comprados, trocados ou beneficiados [10].
A verificação de quanto da disponibilidade de alimentos para consumo, no domicilio, supre a necessidade da família foi elaborada realizando-se o cálculo de energia diária disponível considerando as calorias fornecidas pelos alimentos presentes no domicílio e a soma da necessidade estimada de energia (EER) de todos os membros da família, de acordo com a metodologia proposta pela FAO apresentada por Smith [11]: para cada domicilio, as quantidades de cada item alimentar foram convertidas em quilocalorias utilizando as tabelas de conversão "Tabela para Avaliação do Consumo Alimentar em Medidas Caseiras (5 ed.,2004)" [12] e "Tabela Brasileira de Composição de Alimentos ( ${ }^{\mathrm{a}}$ ed., 2006)" [13], estes valores foram somados e divididos por 30, referente ao número de dias de um mês. Este valor foi dividido pelo número de pessoas residentes no domicílio, a fim de avaliar a suficiência de energia disponível para atender as necessidades alimentares de todos os membros da família, o que possibilitou a classificação das famílias quanto à situação de Segurança ou Insegurança Alimentar.

$\mathrm{O}$ aporte de nutrientes na disponibilidade alimentar considerou a contribuição de carboidratos, proteínas e lipídios (ácidos graxos monoinsaturados, poliinsaturados e saturados) e a participação relativa da disponibilidade alimentar familiar foi expressa a partir do percentual de calorias que o macronutriente representava no total de calorias disponíveis para consumo.

A análise da importância dos gêneros produzidos na disponibilidade dos alimentos se deu por comparação do total de calorias disponíveis com o quanto desta advém da produção [14].

\section{Avaliação do estado nutricional}

O estado nutricional de todos os moradores dos domicílios constou de aferições de peso e altura para determinação do índice de massa corporal (IMC). Utilizou-se para a classificação do estado nutricional de crianças, adolescentes, adultos e idosos, os critérios de IMC propostos pela WHO [15,16,17,18]. Os dados antropométricos foram utilizados para o cálculo da necessidade estimada de energia (EER), a partir de equações para predição do gasto energético total definido a cada faixa etária, para manter a saúde, promover ótimo crescimento e maturação em menores de 20 anos, bem como para garantir nível desejável de atividade física [19], complementando a classificação da situação de Segurança Alimentar e Nutricional. 


\section{Análise dos dados}

Foi criado um banco de dados no programa Microsoft Office Excel 2003 para tabulação das respostas obtidas nos questionários. Utilizou-se o programa DietPro versão 5.1., para a elaboração dos cálculos de calorias fornecidas pelos alimentos disponíveis no domicilio, bem como a porcentagem de carboidrato, proteína e lipídio, e posteriormente estimou-se a necessidade energética individual com as fórmulas de Estimated Energy Requerements (EER) do Institute of Medicine ${ }^{[19]}$.

As análises estatísticas foram feitas com o auxílio dos programas Epi info 6.04 e Sigma-Statistic ${ }^{\circledR}$ for Windows. Foi empregado o teste de Kruskal-Wallis, sendo complementado pelo Teste de Comparações Múltiplas de Dunn's, e o nível de significância do teste $p<0,05$ [20].

\section{Aspectos éticos}

Os entrevistados participaram voluntariamente da coleta de dados, tendo assinado Termo de Consentimento Livre Esclarecido. Este trabalho foi aprovado pelo Comitê de Ética em Pesquisa com Seres Humanos da Universidade Federal de Viçosa sob o no $122 / 2009$.

\section{RESULTADOS E DISCUSSÃO}

A amostra foi constituída de 10 domicílios da zona rural, onde totalizaram 40 moradores. A Tabela 1 sumariza as características da população. Verifica-se discreto predomínio de homens (52,5\%). A maioria $(62,5 \%)$ dos indivíduos integrava o estrato etário de $\geq 20$ e $<60$ (anos) e a prevalência de solteiros foi de $52,5 \%$ e de casados $45,5 \%$.

Tabela 1. Distribuição dos moradores da zona rural de Minas Gerais, segundo gênero, idade e estado civil. São Miguel do Anta, MG, 2010.

\begin{tabular}{lcc}
\hline \multicolumn{1}{c}{ Dados } & $\mathbf{n}$ & $\mathbf{\%}$ \\
\hline Gênero & & \\
$\quad$ Masculino & 21 & 52,50 \\
$\quad$ Feminino & 19 & 47,50 \\
Idade (anos) & & \\
$<10$ & 04 & 10,00 \\
$\geq 10$ e $<20$ & 10 & 25,00 \\
$\geq 20$ e $<60$ & 25 & 62,50 \\
$\geq 60$ & 01 & 2,50 \\
Estado civil & & \\
$\quad$ Solteiro & 21 & 52,50 \\
Casado & 18 & 45,50 \\
$\quad$ Viúvo & 01 & 2,50 \\
\hline Total & $\mathbf{4 0}$ & $\mathbf{1 0 0 , 0 0}$ \\
\hline
\end{tabular}

$\mathrm{Na}$ população estudada, encontrou-se um EER médio de $2487,5 \pm 176,6$ kcal e média da

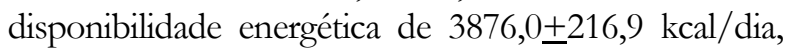
quantidade que supera a necessidade estimada para a totalidade dos indivíduos (Tabela 2). De acordo com o método apresentado por Smith [11], todos os indivíduos foram classificados em situação de Segurança Alimentar. 
Tabela 2. Necessidade calórica e disponibilidade média, desvio-padrão, mediana, mínimo e máximo dos macronutrientes analisados. São Miguel do Anta, MG, 2010.

\begin{tabular}{|c|c|c|c|c|c|}
\hline & Variáveis & Média \pm DP & Mediana & Mínimo & Máximo \\
\hline \multirow{5}{*}{$\begin{array}{l}\frac{0}{0} \\
\frac{\pi}{0} \\
: \frac{0}{0} \\
\frac{0}{0} \\
: 0 \\
: 0\end{array}$} & Necessidade calórica (kcal) & $2487,4 \pm 176,6$ & 2336,0 & 2112,6 & 2597,0 \\
\hline & Energia (kcal) & $3876,0 \pm 216,9$ & 4162,1 & 2254,1 & 5658,8 \\
\hline & Proteína (g) & $111,0 \pm 37,4$ & 106,9 & 59,1 & 155,0 \\
\hline & Lipídeo (g) & $165,4 \pm 25,6$ & 187,8 & 61,2 & 238,8 \\
\hline & Carboidrato (g) & $474,9 \pm 34,0$ & 479,1 & 342,3 & 650,1 \\
\hline
\end{tabular}

Apesar da população em estudo estar classificada em situação de segurança alimentar há de se considerar que o excesso de energia disponível associado à densidade energética dos alimentos constituídos por gorduras e açucares, e ao aumento de sedentarismo e diminuição de ambientes que oferecem oportunidades para a atividade física podem levar a obesidade [21].

Considerando diferentes abordagens metodológicas, em 1962 a Fundação Getulio Vargas [22] estudou a disponibilidade calórica per capita utilizando inquérito de consumo alimentar no Brasil e considerou como necessidade mínima a ingestão de $2.450 \mathrm{kcal} /$ dia. A Pesquisa de Orçamento Familiar 2008-2009 [23] apresentou como resultado da disponibilidade domiciliar média de alimentos 1.536 $\mathrm{kcal} / \mathrm{pessoa} / \mathrm{dia}$ no meio urbano e de 1973 $\mathrm{kcal} / \mathrm{pessoa} /$ dia no rural. Delgado [24] afirma que a produção nacional se encontrava na faixa de 3.000 $\mathrm{kcal} / \mathrm{pessoa} /$ dia. Estes valores estão abaixo do encontrado neste estudo, que identificou disponibilidade calórica de $156 \%$ da necessidade energética média $(2487,4 \pm 176,6)$.

Em contrapartida, dados da Pesquisa Nacional por Amostra de Domicílio, de 2006 [25], revelam que no meio rural encontra-se a maior prevalência domiciliar de Insegurança Alimentar moderada ou grave ao utilizar a Escala Brasileira de Insegurança Alimentar (EBIA), método subjetivo de mensuração da SAN. Hoffmann [26] ao analisar dados da Pesquisa Nacional por Amostras de Domicílios (PNAD) de 2004 encontrou prevalência de 34,9\% dos domicílios com algum grau de insegurança alimentar pela EBIA e segundo o autor, o determinante isolado desta insegurança mais importante é a renda domiciliar per capita e quando comparadas áreas urbanas e rurais, a insegurança alimentar é maior nas últimas. Mas, quando é controlado o efeito da renda e da escolaridade da pessoa de referência do domićlio, verifica-se que a insegurança alimentar tende a ser menor nas áreas rurais. Fenômeno semelhante ocorre na análise do efeito de o setor de atividade da pessoa de referência ser ou não agrícola.

Neste estudo, do total de calorias disponíveis $40,5 \%$ provinham da produção familiar e o restante fornecido pelos alimentos adquiridos por meio de compra monetária. A Tabela 3 apresenta a participação relativa de macronutrientes na disponibilidade alimentar domiciliar indicando que 50,6\% das calorias totais provinham de carboidratos, $11,52 \%$ de proteínas e 37,88\% de lipídeos. 
Tabela 3. Participação dos macronutrientes, da sacarose e da composição lipídica no total de calorias. São Miguel do Anta, MG, 2010.

\begin{tabular}{lccc}
\hline \multicolumn{1}{c}{ Macronutrientes } & \% de adequação calórica & \multicolumn{2}{c}{ \% da participação } \\
\cline { 3 - 4 } & & Compra & Produção \\
\hline Carboidratos & 50,6 & 70,9 & 29,1 \\
$\quad$ Açúcar (sacarose) & 16,5 & 100,0 & - \\
Proténas & 11,5 & 47,0 & 54,0 \\
Lipídeos & 37,9 & 44,0 & 56,0 \\
$\quad$ Ácidos graxos monoinssaturados & 12,9 & 30,5 & 69,5 \\
$\quad$ Ácidos graxos poliinsaturados & 9,9 & 63,4 & 36,6 \\
$\quad$ Ácidos graxos saturados & 11,8 & 31,3 & 68,7 \\
\hline
\end{tabular}

Os resultados deste estudo evidenciam adequação da disponibilidade às recomendações nutricionais de carboidrato e proteína e inadequação de lipídeos de acordo com o preconizado pela Acceptable Macronutrients Distribuition Range (AMDR) do Institute of Medicine (2002) ${ }^{[19]}$. A inadequação dos lipídeos veio acompanhada de alto teor de ácidos graxos saturados, estando estes acima de 10\% das calorias totais. Apesar da adequação dos carboidratos há excesso relativo da fração de sacarose que representa $16,52 \%$ das calorias totais quando o máximo fixado pela Organização Mundial da Saúde [27] é de 10\%. Tais valores são divididos de acordo com sua origem de aquisição, o que revela maior gasto mensal com a compra de carboidratos $(70,9 \%)$, principalmente sacarose, e elevada disponibilidade de ácidos graxos saturados oriundos, principalmente, da produção animal (68,7\%).

Em estudo semelhante com agricultores familiares Troian ${ }^{[28]}$ relatou a existência de elevado consumo de alimentos energéticos, carboidratos consumidos em excesso e insuficiências no consumo de frutas e hortaliças. Na Pesquisa de Orçamento Familiar 2008-2009 [23] a participação relativa de macronutrientes na disponibilidade alimentar é de
$59 \%$ de carboidratos, $12 \%$ de proteínas e $29 \%$ de lipídeos, evidenciando adequação da disponibilidade de alimentos dos brasileiros às recomendações nutricionais.

Os agricultores deste estudo produzem e consomem alimentos desta produção, mas também transacionam no mercado estes produtos agrícolas e não-agrícolas, para conseguir comprar o necessário para complementar a sua alimentação. Isso é necessário devido a não produção da totalidade dos produtos e mercadorias consumidas, a sazonalidade da produção agrícola, a não possibilidade de armazenamento de alguns gêneros alimentícios, a imprevistos climáticos que afetam a produção e ao desejo do agricultor em produzir sua própria alimentação.

Neste estudo, do total de quarenta pessoas, $62,5 \%$ foram classificados como eutróficos, $22,5 \%$ em sobrepeso, $7,5 \%$ obesas e $7,5 \%$ em baixo peso, sendo que as mulheres com mais de 20 anos apresentavam a maioria dos casos de inadequação de excesso de peso e as crianças responsáveis por $50 \%$ dos casos de baixo peso (Figura 1). 
Figura 1. Distribuição dos indivíduos da amostra de acordo com o estado nutricional. São Miguel do Anta MG, 2010.

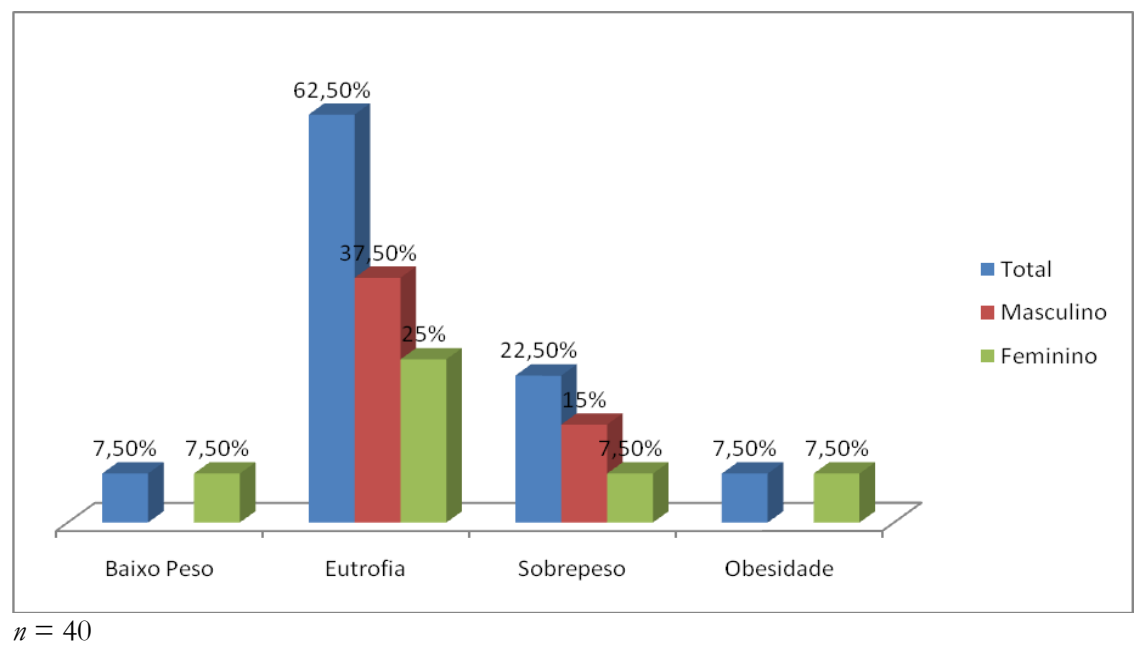

Marinho [29] relata que tradicionalmente, as atividades da mulher da zona rural são voltadas ao cultivo de hortas no entorno da residência e ao serviço doméstico em residências que têm geralmente poucos cômodos. Nessas condições, a dona de casa tem pouco espaço para a atividade física. Assim, a vida sedentária da mulher rural e a alimentação rica em calorias devem influir no fator de risco para a obesidade encontrada nesse grupo.

Outro fator preocupante reside no futuro das crianças com baixo peso, pois em estudo comparativo entre crianças com diagnóstico de baixa estatura e eutróficas, com idade entre 8 e 11 anos, residentes em favelas no Município de São Paulo, verificou alterações metabólicas quanto à redução na oxidação lipídica no período pós-prandial, nas crianças com baixa estatura, aspecto que poderia favorecer acúmulo de gordura corporal neste grupo, mostrando consistência à teoria de que a desnutrição no início da vida acarretaria modificações metabólicas permanentes [30].

Semelhante aos resultados encontrados neste estudo, dados da POF 2008-2009 [31] revelaram que o excesso de peso atingiu cerca de metade dos homens e das mulheres no Brasil, sendo obesos na zona rural $8,8 \%$ e $16,5 \%$ respectivamente e em ambos aumentava a frequência com o aumento da idade para depois declinarem após 60 anos. Entre homens a prevalência do excesso de peso é maior nas áreas urbanas e entre mulheres, nas rurais.

A disponibilidade média de energia e de macronutrientes, segundo estado nutricional dos indivíduos das famílias estudadas não apresentou diferença significativa, mas notou-se que esta apresentou valores mais altos para o grupo com excesso de peso e menores para baixo peso (Tabela 4).

Tabela 4. Disponibilidade média e desvio-padrão de energia e de macronutrientes, segundo estado nutricional dos indivíduos das famílias estudadas. São Miguel do Anta - MG, abril - maio de 2010.

\begin{tabular}{lccc}
\hline \multicolumn{1}{c}{ Variáveis } & Baixo Peso & Eutrofia & Excesso de peso \\
\hline Energia $(\mathrm{kcal})$ & $3795 \pm 1160,5$ & $3862,4 \pm 935,1$ & $4019,9 \pm 909$ \\
Carboidrato $(\mathrm{g})$ & $480,7 \pm 116,6$ & $468,7 \pm 76,7$ & $484,9 \pm 84,4$ \\
Proteína $(\mathrm{g})$ & $11,6 \pm 41,5$ & $119,7 \pm 33,9$ & $100,2 \pm 27,8$ \\
Lipídeo $(\mathrm{g})$ & $58,3 \pm 58,6$ & $163,4 \pm 60,2$ & $186,3 \pm 50,5$ \\
\hline
\end{tabular}


A disponibilidade de alimentos não corresponde ao consumo, mas possibilita inferências, assim alguns autores, estudando ingestão alimentar e o excesso de peso, encontraram relação entre maior ingestão energética, protéica e de lipídeos e aumento de parâmetros antropométricos que refletem a gordura corporal, outros encontraram relação apenas energética e de lipídeos ${ }^{32,33] .}$

Segundo Freitas [32] o hábito alimentar é compreendido na dimensão do cotidiano, em que a experiência faz parte da identidade cultural do sujeito. E para Marinho [29] e Faria [33] embora o padrão alimentar da população rural tenha sofrido poucas mudanças em relação à dieta do agricultor tradicional, baseada na ingestão diária de arroz, feijão e verduras, sendo o consumo de carne apenas ocasional, estes têm apresentado mudanças decorrentes do maior consumo de alimentos industrializados, em substituição às tradicionais comidas caseiras. Estas transformações permanecem, no entanto acompanhadas de hábitos antigos como o consumo da gordura de porco.

\section{CONCLUSÃO}

Neste estudo todas as famílias foram classificadas em situação de Segurança Alimentar e Nutricional de acordo com a disponibilidade calórica encontrada. Porém, o excesso de energia disponível deve ser levado em consideração, assim como a distribuição dos macronutrientes que mostrou elevada disponibilidade domiciliar de lipídeos $(37,88 \%)$ e do total de calorias disponíveis, apenas 40,5\% era proveniente da produção, sendo o gasto mensal maior com carboidratos, principalmente sacarose.

Assim, apesar da classificação, pela FAO, de Segurança Alimentar e Nutricional, de acordo com a disponibilidade calórica que possuíam, e da predominância do estado de eutrofia, a qualidade da dieta desta população não apresenta a adequação esperada e a origem dos alimentos disponíveis mostrase vulnerável a valores monetários, além de aumentar a presença de produtos industrializados.

Acredita-se que a produção para autoconsumo seja capaz de gerar princípios da Segurança Alimentar e Nutricional como o acesso e disponibilidade dos alimentos a serem consumidos, a qualidade nutricional destes, bem como o fornecimento das quantidades suficientes $\mathrm{e}$ permanentes de alimentos e o abastecimento das famílias com uma alimentação que atenda aos hábitos culturais. Porém, devido à produção de alimentos para o autoconsumo das famílias serem mercantilizada e vulnerabilizada pelas opções produtivas relativas à especialização produtiva, as famílias rurais tornaram-se mais propícias a apresentarem situação de Insegurança Alimentar e Nutricional.

\section{AGRADECIMENTO}

À equipe do projeto "Apoio à transição agroecológica de novas famílias no território da Serra do Brigadeiro, Minas Gerais" do Departamento de Fitotecnia da Universidade Federal de Viçosa, à EMATER - MG de São Miguel do Anta e a todas as famílias pelo apoio e colaboração.

\section{REFERÊNCIAS}

[1] Silva JRS. Segurança alimentar, produção agrícola familiar e assentamentos de reforma agrária no Maranhão [dissertação]. São Luis: Universidade Federal do Maranhão; 2006 .

[2] Food and Agriculture Organization - FAO. Programa Alimentar Mundial (PAM): Relatório 2010. Roma; 2010.

[3] Brasil. Decreto no 5.079, de 12 de maio de 2004. Tem por finalidade propor a formulação de políticas públicas e diretrizes para a Política Nacional de Segurança Alimentar e Nutricional do Governo Federal. Diário Oficial da União. 13 maio 2004.

[4] Maluf RS. Segurança alimentar e fome no Brasil - 10 anos da Cúpula Mundial de Alimentação: relatório técnico no 2 CERESAN. Rio de Janeiro; 2006.

[5] Belik W. Perspectivas para segurança alimentar e nutricional no Brasil. Saúde Soc. 2003;12(1):12-20.

[6] Vogt SPC, Souza RS. Políticas públicas e segurança alimentar: estudo de caso do programa de compra antecipada especial com doação simultânea (Fome Zero) no município de tenente Portela - RS. In: Livro de Resumos do VII Congresso Brasileiro de Sistemas de Produção Agricultura Familiar, Políticas Públicas e Inclusão Social. Londrina; 2007.

[7] Gazolla M. Agricultura familiar, segurança alimentar e políticas publicas: uma análise a partir da produção para autoconsumo no território do Alto Uruguai/RS [dissertação]. Porto Alegre: Universidade Federal do Rio Grande do Sul; 2004. 
[8] IBGE - Instituto Brasileiro de Geografia e Estatística. Censo Agropecuário 2006. Agricultura Familiar. Primeiros Resultados. Brasil, Grandes Regiões e Unidades da Federação. MDA/MPOG. Rio de Janeiro; 2009.

[9] IBGE - Instituto Brasileiro de Geografia e Estatística [internet]. Rio de Janeiro; 2009 [acesso em 15 mar 2009]. Disponível em http://www.ibge.gov.br/cidadesat

[10] Anjos LA, Souza DR, Rossato SL. Desafios na medição quantitativa da ingestão alimentar em estudos populacionais. Rev. Nutr. 2009;22(1):151-161.

[11] Smith LC. The use of household expenditure surveys for the assessment of food insecurity. In: Proceedings Measurement and assessment of food deprivation and undernutrition. International Scientific Symposium. Roma; 2002.

[12] Pinheiro ABV, Neves PA, Lacerda EMA, Benzecry EH, Gomes MCS, Costa VM. Tabela para Avaliação de Consumo Alimentar em Medidas Caseiras. 5.ed. São Paulo: Editora Atheneu; 2004.

[13] Núcleo de Estudos e Pesquisas em Alimentação. Universidade Estadual de Campinas [NEPA/Unicamp]. Tabela Brasileira de Composição de Alimentos [TACO]: versão 2. São Paulo: NEPA/Unicamp; 2006.

[14] Levy-Costa RB, Sichieri R, Pontes NS, Monteiro CA. Disponibilidade domiciliar de alimentos no Brasil: distribuição e evolução (1974-2003). Rev Saúde Pública. 2005;39(4):530-540.

[15] World Health Organization. Physical Status: the use and interpretation of anthropometry. WHO Technical Report Series no 854. Geneva: WHO; 1995.

[16] World Health Organization. Obesity: Preventing and managing the global epidemic - Report of a WHO consultation on obesity. WHO Technical Report Series no 894. Geneva WHO; 2000.

[17] Onis. M, Onyango AW, Borghi E, Siyam A, Nishida C, Siekmann J. Development of a WHO growth reference for school-aged children and adolescents. Bulletin of the World Health Organization; 2007.

[18] World Health Organization. WHO Child Growth Standards: Length/height-for-age, weight-for-age, weightfor-length, weight-for-height and body mass index-for-age. Methods and development. WHO (nonserial publication). Geneva: WHO; 2006.

[19] Institute of Medicine. In: Dietary References Intakes of energy, carboidrate, fiver, fat, fatty acids, cholesterol, protein and amino acids. Washington, DC: The National Academy Press; 2002.

[20] Callegari-Jacques SM. Bioestatística: princípios e aplicações. Porto Alegre: Artmed; 2003.

[21] International Association for the Study of Obesity (2002). Obesity in Europe: the case for action [internet]. [acesso em 23 maio 2012]. Disponível em: http://www.iotf.org/media/obesity.pdf.

[22] Fundação Getúlio Vargas. U.S. Department of Agriculture. Economic Research Service. Food consumption in Brazil: family budget surveys in the early 1960's. Jerusalem: Keter Press; 1970.

[23] IBGE - Instituto Brasileiro de Geografia e Estatística. Pesquisa de Orçamentos Familiares - POF - 2008-2009 Aquisição alimentar domiciliar per capita - Brasil e Grandes Regiões. Rio de Janeiro; 2010.

[24] Delgado G. Segurança alimentar e desenvolvimento rural - o Plano Safra 2003-2004 e o Plano Nacional de Reforma Agrária. In: Rocha M (org). Segurança alimentar um desafio para acabar com a fome no Brasil. São Paulo: Ed. Fundação Perseu Abramo; 2004.

[25] IBGE - Instituto Brasileiro de Geografia e Estatística. Pesquisa Nacional por Amostra de Domicilios - PNAD Segurança Alimentar - 2004. Rio de Janeiro; 2006.

[26] Hoffmann, R. Determinantes da Insegurança Alimentar no Brasil: Análise dos Dados da PNAD de 2004. Segurança Alimentar e Nutricional. 2008;15(1):49-61.

[27] World Health Organization. Diet, Nutrition and the prevention of chronic diseases. WHO Technical Report Series no 916. Geneva: WHO; 2003.

[28] Troian A, Troian A, Dalcin D, Oliveira SV. Agricultura familiar e autoconsumo: um estudo no município de Encantado, RS. In: Seminario Taller de la Red SIAL. Argentina; 2009.

[29] Marinho SP, Martins IS, Perestrelo JPP, Oliveira DC. Obesidade em adultos de segmentos pauperizados da sociedade. Rev. Nutr. 2003;16(2):195-201.

[30] Hoffman DJ, Sawaya AL, Verreschi I, Tucker KL, Roberts SB. Why are nutritionally stunted children at increased risk of obesity? Studies of metabolic rate and fat oxidation in shantytown children from São Paulo, Brazil. Am J Clin Nutr. 2000;72:702-707.

[29] IBGE - Instituto Brasileiro de Geografia e Estatística. Pesquisa de Orçamentos Familiares - POF - 2008-2009 - 
Antropometria e estado nutricional de crianças, adolescentes e adultos no Brasil. Rio de Janeiro; 2010.

[30] Miller WC, Lindeman AK, Wallace J, Niederpruem M. Diet composition, energy intake, and exercise in relation to body fat in men and women. Am J Clin Nutr. 1990;52:426430.

[31] Tucker LA, Kano MJ. Dietary fat and body fat: a multivariate study of 205 adult females. Am J Clin Nutr. 1992:56:616-622.
[32] Freitas MCS, Pena PGL. Segurança alimentar e nutricional: a produção do conhecimento com ênfase nos aspectos da cultura. Rev. Nutr. 2007;20(1):69-81.

[33] Faria ER. Critérios diagnósticos e fatores de risco para síndrome metabólica, em adolescentes que já apresentaram a menarca, de escolas públicas de Viçosa - MG [dissertação]. Viçosa: Universidade Federal de Viçosa; 2007. 